\title{
A Multiparametric Quon Algebra
}

\author{
Hery Randriamaro *
}

September 16, 2019

\begin{abstract}
The quon algebra is an approach to particle statistics introduced by Greenberg in order to provide a theory in which the Pauli exclusion principle and Bose statistics are violated by a small amount. We generalize these models by introducing a deformation of the quon algebra generated by a collection of operators $\mathrm{a}_{i}, i \in \mathbb{N}^{*}$ the set of positive integers, on an infinite dimensional module satisfying the $q_{i, j}$-mutator relations $\mathrm{a}_{i} \mathrm{a}_{j}^{\dagger}-q_{i, j} \mathrm{a}_{j}^{\dagger} \mathrm{a}_{i}=\delta_{i, j}$. The realizability of our model is proved by means of the Aguiar-Mahajan bilinear form on the chambers of hyperplane arrangements. We show that, for suitable values of $q_{i, j}$, the module generated by the particle states obtained by applying combinations of $\mathrm{a}_{i}$ 's and $a_{i}^{\dagger}$ 's to a vacuum state $|0\rangle$ is an indefinite Hilbert module. Furthermore, we refind the extended Zagier's conjecture established independently by Meljanac et al. and by Duchamp et al.
\end{abstract}

Keywords: Quon Algebra, Indefinite Hilbert Module, Hyperplane Arrangement MSC Number: 05E15, 81R10

\section{Introduction}

Denote by $\mathbb{C}\left[q_{i, j}\right]$ the polynomial ring $\mathbb{C}\left[q_{i, j} \mid i, j \in \mathbb{N}^{*}\right]$ with variables $q_{i, j}$. The quons are particles whose annihilation and creation operators obey the quon algebra which interpolates between fermions and bosons.

Definition 1.1. By multiparametric quon algebra is meant the free algebra $\mathbf{A}$, equal to $\mathbb{C}\left[q_{i, j}\right]\left[\mathbf{a}_{i} \mid i \in \mathbb{N}^{*}\right]$, and subject to the anti-involution $\dagger$ exchanging $\mathbf{a}_{i}$ with $\mathbf{a}_{i}^{\dagger}$ and to the commutation relations

$$
\mathrm{a}_{i} \mathrm{a}_{j}^{\dagger}=q_{i, j} \mathrm{a}_{j}^{\dagger} \mathrm{a}_{i}+\delta_{i, j}
$$

where $\delta_{i, j}$ is the Kronecker delta.

The multiparametric quon algebra is a generalization of the deformed quon algebra subject to the restriction $q_{i, j}=\bar{q}_{j, i}$ independently studied by Bozejko and Speicher [2, $\left.\S 3\right]$, and by Meljanac and Svrtan [6, $\S 1.1]$. Their algebra is in turn a generalization of the deformed quon algebra investigated by Speicher subject to the restriction $q_{i, j}=q_{j, i}[8]$. Then, his algebra is a generalization of the quon algebra introduced by Greenberg [5] and studied by Zagier

${ }^{*}$ This research was supported by my mother

Lot II B 32 bis Faravohitra, 101 Antananarivo, Madagascar

e-mail: hery.randriamaro@gmail.com 
[9, $\S 1$ 1] which is subject to the commutation relations $\mathrm{a}_{i} \mathrm{a}_{j}^{\dagger}=q \mathrm{a}_{j}^{\dagger} \mathrm{a}_{i}+\delta_{i, j}$ obeyed by the annihilation and creation operators of the quon particles, and generating a model of infinite statistics. Finally, the quon algebra is a generalization of the classical Bose and Fermi algebras corresponding to the restrictions $q=1$ and $q=-1$ respectively, as well as of the intermediate case $q=0$ suggested by Hegstrom and investigated by Greenberg [4].

In a Fock-like representation, the generators of $\mathbf{A}$ are the linear operators $\mathrm{a}_{i}, \mathrm{a}_{i}^{\dagger}: \mathbf{V} \rightarrow \mathbf{V}$ on an infinite dimensional $\mathbb{C}\left[q_{i, j}\right]$-vector module $\mathbf{V}$ satisfying the commutation relations

$$
\mathrm{a}_{i} \mathrm{a}_{j}^{\dagger}=q_{i, j} \mathrm{a}_{j}^{\dagger} \mathrm{a}_{i}+\delta_{i, j}
$$

and the relations

$$
\mathrm{a}_{i}|0\rangle=0,
$$

where $\mathrm{a}_{i}^{\dagger}$ is the adjoint of $\mathrm{a}_{i}$, and $|0\rangle$ is a nonzero distinguished vector of $\mathbf{V}$. The $\mathbf{a}_{i}$ 's are the annihilation operators and the $\mathrm{a}_{i}^{\dagger}$ 's the creation operators.

Define the $q_{i, j}$-conjugate $\tilde{P}$ of a monomial $P=\mu \prod_{u \in[n]} q_{i_{u}, j_{u}} \in \mathbb{C}\left[q_{i, j}\right]$, where $\mu \in \mathbb{C}$, by

$$
\tilde{P}:=\tilde{\mu} \prod_{s \in[n]} \tilde{q}_{i_{s}, j_{s}} \quad \text { with } \quad \tilde{\mu}=\bar{\mu}, \text { and } \tilde{q}_{i_{s}, j_{s}}=q_{j_{s}, i_{s}}
$$

and the $q_{i, j}$-conjugate of a monomial sum $Q=P_{1}+\cdots+P_{k} \in \mathbb{C}\left[q_{i, j}\right]$ by $\tilde{Q}=\tilde{P}_{1}+\cdots+\tilde{P}_{k}$.

Definition 1.2. An indefinite inner product on $\mathbf{V}$ is a map $(.,):. \mathbf{V} \times \mathbf{V} \rightarrow \mathbb{C}\left[q_{i, j}\right]$ such that, for $\mu \in \mathbb{C}$, and $\mathrm{u}, \mathrm{v}, \mathrm{w} \in \mathbf{V}$, we have

- $(\mu \mathrm{u}, \mathrm{v})=\mu(\mathrm{u}, \mathrm{v})$ and $(\mathrm{u}+\mathrm{v}, \mathrm{w})=(\mathrm{u}, \mathrm{w})+(\mathrm{v}, \mathrm{w})$,

- $(\mathrm{u}, \mathrm{v})=\widetilde{(\mathrm{v}, \mathrm{u})}$,

- and, if $\mathrm{u} \neq 0,(\mathrm{u}, \mathrm{u}) \in \mathbb{C}\left[q_{i, j}\right] \backslash\{0\}$.

Let $\mathbf{H}$ be the submodule of $\mathbf{V}$ generated by the particle states obtained by applying combinations of $\mathrm{a}_{i}$ 's and $\mathbf{a}_{i}^{\dagger}$ 's to $|0\rangle$, that is $\mathbf{H}:=\{a|0\rangle \mid \mathrm{a} \in \mathbf{A}\}$. The aim of this article is to prove the realizability of that model through the following theorem.

Theorem 1.3. Under the condition $\left|q_{i, j}\right|<1$, the module $\mathbf{H}$ is an indefinite Hilbert module for the map (.,.) : $\mathbf{H} \times \mathbf{H} \rightarrow \mathbb{C}\left[q_{i, j}\right]$ defined, for $\mu, \nu \in \mathbb{C}\left[q_{i, j}\right]$, and $\mathrm{a}, \mathrm{b} \in \mathbf{A}$, by

$$
(\mu \mathrm{a}|0\rangle, \nu \mathrm{b}|0\rangle):=\mu \tilde{\nu}\left\langle 0\left|\mathrm{ab}^{\dagger}\right| 0\right\rangle \quad \text { with } \quad\langle 0 \mid 0\rangle=1,
$$

and where the usual bra-ket product $\left\langle 0\left|\mathrm{a} \mathrm{b}^{\dagger}\right| 0\right\rangle$ is subject to the defining relations of $\mathbf{A}$.

The indefinite inner product of Theorem 1.3 becomes an inner product when the matrix representing (.,.) is positively diagonalizable. Theorem 1.3 is particularly a generalization of the realizability of the deformed quon algebra model for $q_{i, j}=\bar{q}_{j, i}$ established independently by Bozejko and Speicher [2, Corollary 3.2], and by Meljanac and Svrtan [6, Theorem 1.9.4], which in turn is a generalization of the realizability of the deformed quon algebra model for $q_{i, j}=q_{j, i}$ established by Speicher [8, Corollary], which finally is a generalization of the realizability of the quon algebra model established by Zagier [9, Theorem 1]. 
To prove Theorem 1.3 , we first show with Lemma 3.1 that

$$
\mathbf{B}:=\left\{\mathbf{a}_{i_{1}}^{\dagger} \ldots \mathbf{a}_{i_{n}}^{\dagger}|0\rangle \mid\left(i_{1}, \ldots, i_{n}\right) \in\left(\mathbb{N}^{*}\right)^{n}, n \in \mathbb{N}\right\}
$$

is a basis of $\mathbf{H}$, so that we can assume that $\mathbf{H}=\left\{\sum_{i=1}^{n} \mu_{i} \mathrm{~b}_{i} \mid n \in \mathbb{N}^{*}, \mu_{i} \in \mathbb{C}\left[q_{i, j}\right], \mathrm{b}_{i} \in \mathbf{B}\right\}$. The infinite matrix associated to the map of Theorem 1.3 is $\mathbf{M}:=((\mathrm{b}, \mathrm{a}))_{\mathrm{a}, \mathrm{b} \in \mathbf{B}}$. Let $\left(\left(\begin{array}{c}\mathbb{N}^{*} \\ n\end{array}\right)\right)$ be the set of multisets of $n$ elements in $\mathbb{N}^{*}$. We prove with Lemma 3.2 that

$$
\mathbf{M}=\bigoplus_{n \in \mathbb{N}^{*}} \bigoplus_{I \in\left(\left(\begin{array}{c}
\mathbb{N}^{*} \\
n
\end{array}\right)\right)} \mathbf{M}_{I} \quad \text { with } \quad \mathbf{M}_{I}=\left(\left\langle 0\left|\mathrm{a}_{\dot{\tau}(n)} \ldots \mathrm{a}_{\dot{\tau}(1)} \mathrm{a}_{\dot{\sigma}(1)}^{\dagger} \ldots \mathrm{a}_{\dot{\sigma}(n)}^{\dagger}\right| 0\right\rangle\right)_{\dot{\sigma}, \dot{\tau} \in \mathfrak{S}_{I}},
$$

where $\mathfrak{S}_{I}$ is the permutation set of the multiset $I$. For example,

$$
\mathbf{M}_{[3]}=\left(\begin{array}{cccccc}
1 & q_{3,2} & q_{2,1} & q_{2,1} q_{3,1} & q_{3,1} q_{3,2} & q_{3,1} q_{2,1} q_{3,2} \\
q_{2,3} & 1 & q_{2,1} q_{2,3} & q_{2,1} q_{3,1} q_{2,3} & q_{3,1} & q_{3,1} q_{2,1} \\
q_{1,2} & q_{1,2} q_{3,2} & 1 & q_{3,1} & q_{3,2} q_{1,2} q_{3,1} & q_{3,2} q_{3,1} \\
q_{1,2} q_{1,3} & q_{1,2} q_{1,3} q_{3,2} & q_{1,3} & 1 & q_{3,2} q_{1,2} & q_{3,2} \\
q_{1,3} q_{2,3} & q_{1,3} & q_{2,3} q_{2,1} q_{1,3} & q_{2,3} q_{2,1} & 1 & q_{2,1} \\
q_{1,3} q_{1,2} q_{2,3} & q_{1,3} q_{1,2} & q_{2,3} q_{1,3} & q_{2,3} & q_{1,2} & 1
\end{array}\right) .
$$

Proposition 2.1 and Lemma 3.3 permits us to deduce that, if $J \in\left(\begin{array}{c}\mathbb{N}^{*} \\ n\end{array}\right) \subseteq\left(\left(\begin{array}{c}\mathbb{N}^{*} \\ n\end{array}\right)\right)$, then

$$
\operatorname{det} \mathbf{M}_{J}=\prod_{\substack{K \in 2^{J} \\
\# K^{J}}}\left(1-\prod_{\{s, t\} \in\left(\begin{array}{c}
K \\
2
\end{array}\right)} q_{s, t} q_{t, s}\right)^{(\# K-2) !(n-\# K+1) !} .
$$

For example, $\operatorname{det} \mathbf{M}_{[3]}=\left(1-q_{1,2} q_{2,1}\right)^{2}\left(1-q_{1,3} q_{3,1}\right)^{2}\left(1-q_{2,3} q_{3,2}\right)^{2}\left(1-q_{1,2} q_{2,1} q_{1,3} q_{3,1} q_{2,3} q_{3,2}\right)$. That determinant was independently computed by Meljanac and Svrtan for the specialization $q_{i, j}=\bar{q}_{j, i}$ [6. Theorem 1.9.2], by Duchamp et al. for the specialization $q_{i, j}=q_{j, i}[\underline{3}, \S$ 6.4.1], and by Zagier for the specialization $q_{i, j}=q_{j, i}=q$ [9, Theorem 2].

Moreover, consider the multiset $I=\{\overbrace{i_{1}, \ldots, i_{1}}^{p_{1} \text { times }}, \overbrace{i_{2}, \ldots, i_{2}}^{p_{2} \text { times }}, \ldots, \overbrace{k, \ldots, k}^{p_{k} \text { times }}\} \in\left(\left(\begin{array}{c}\mathbb{N}^{*} \\ n\end{array}\right)\right)$. For $s \in[n]$, let $\dot{s}:=i_{j}$ if $s \in\left[p_{j}+p_{j-1}+\cdots+p_{1}\right] \backslash\left[p_{j-1}+\cdots+p_{1}\right]$. Suppose that the generators of $\mathbf{A}$ satisfy the commutation relations $\mathrm{a}_{s} \mathrm{a}_{t}^{\dagger}=q_{\dot{s}, \dot{t}} \mathrm{a}_{t}^{\dagger} \mathrm{a}_{s}+\delta_{s, t}$. In that case, if we regard $\mathbf{M}_{[n]}$ as the matrix representing a linear map $\alpha: M \rightarrow M$ on a module $M$, then we prove with Proposition 2.2 and Lemma 3.4 that $\mathbf{M}_{I}$ is the matrix representing $\alpha$ restricted to a submodule $N \subseteq M$ such that $\alpha(N)=N$. Therefore, we can infer that, for every $I \in\left(\left(\begin{array}{c}\mathbb{N}^{*} \\ n\end{array}\right)\right), \mathbf{M}_{I}$ is nonsingular for $\left|q_{i, j}\right|<1, i, j \in \mathbb{N}^{*}$.

When, for special values of the $q_{i, j}$ 's, $\mathbf{M}_{[n]}$ is diagonalizable, then $\mathbf{M}_{I}$ becomes positive definite. Indeed, as $\mathbf{M}_{I}$ is the identity matrix if $q_{i, j}=0$, for every $i, j \in \mathbb{N}^{*}$, we deduce by continuity that $\mathbf{M}_{I}$ is positive definite. For these suitable values of $q_{i, j}, \mathbf{M}$ becomes a positive definite matrix or, in other terms, the map in Theorem 1.3 becomes an inner product on $\mathbf{H}$. It is 
the case of the algebras investigated by Meljanac and Svrtan, and Zagier since, with their models, $\mathbf{M}_{[n]}$ is a hermitian matrix, that is consequently diagonalizable.

Finally, we provide another proof of the extended Zagier's conjecture in Section 4

Proposition 1.4. Let $n \in \mathbb{N}^{*}$, and assume that the generators of $\mathbf{A}$ satisfy the commutation relations $\mathrm{a}_{s} \mathrm{a}_{t}^{\dagger}=q \mathrm{a}_{t}^{\dagger} \mathrm{a}_{s}+\delta_{s, t}$. Then, each entry of $\mathbf{M}_{[n]}^{-1}$ is an element of $\frac{\mathbb{C}[q]}{\prod_{i \in[n-1]}\left(1-q^{i^{2}+i}\right)^{n-i}}$.

The extended Zagier's conjecture was first established by Meljanac and Svrtan [6, Corollary 2.2.8] who disproved Zagier's conjecture by using the algorithm of [6, Proposition 2.2.15] for $n=8$. One deduces also Proposition 1.4 from the study of the representation of the permutation group made by Duchamp et al. [3, Proposition 4.6, 4.9].

\section{Hyperplane Arrangements}

We establish two results we need concerning the hyperplane arrangement associated to the permutation group of $n$ elements in order to prove Theorem 1.3 .

Recall that a hyperplane in the space $\mathbb{R}^{n}$ is a $(n-1)$-dimensional linear subspace, and a hyperplane arrangement is a finite set of hyperplanes. For a hyperplane $H$, denote its two associated open half-spaces by $H^{+}$and $H^{-}$, and let $H^{0}:=H$. A face of a hyperplane arrangement $\mathcal{A}$ is a subset of $\mathbb{R}^{n}$ having the form

$$
F:=\bigcap_{H \in \mathcal{A}} H^{\epsilon_{H}(F)} \quad \text { with } \quad \epsilon_{H}(F) \in\{+, 0,-\} .
$$

A chamber of $\mathcal{A}$ is a face $C \in F_{\mathcal{A}}$ such that, for every $H \in \mathcal{A}, \epsilon_{H}(F) \neq 0$. Denote the set formed by the chambers of $\mathcal{A}$ by $C_{\mathcal{A}}$. Assign a variable $h_{H}^{\varepsilon}, \varepsilon \in\{+,-\}$, to every half-space $H^{\varepsilon}$. We work with the polynomial ring $R_{\mathcal{A}}:=\mathbb{Z}\left[h_{H}^{\varepsilon} \mid H \in \mathcal{A}, \varepsilon \in\{+,-\}\right]$, and the module of $R_{\mathcal{A}}$-linear combinations of chambers $M_{\mathcal{A}}:=\left\{\sum_{C \in C_{\mathcal{A}}} x_{C} C \mid x_{C} \in R_{\mathcal{A}}\right\}$.

For $C, D \in C_{\mathcal{A}}$, let $H_{C, D}$ be the set of half-spaces $\left\{H^{\epsilon_{H}(C)} \mid H \in \mathcal{A}, \epsilon_{H}(C)=-\epsilon_{H}(D)\right\}$. The Aguiar-Mahajan bilinear form v $: M_{\mathcal{A}} \times M_{\mathcal{A}} \rightarrow R_{\mathcal{A}}$ is defined, for $C, D \in C_{\mathcal{A}}$, by

$$
\mathrm{v}(C, C)=1 \text { and } \quad \mathrm{v}(C, D)=\prod_{H^{\varepsilon} \in H_{C, D}} h_{H}^{\varepsilon} \text { if } C \neq D .
$$

From $\mathrm{v}$ is defined the linear map $\gamma: M_{\mathcal{A}} \rightarrow M_{\mathcal{A}}$, for $D \in C_{\mathcal{A}}$, by $\gamma(D):=\sum_{C \in C_{\mathcal{A}}} \mathrm{v}(D, C) C$.

Let $x=\left(x_{1}, \ldots, x_{n}\right)$ be a variable of $\mathbb{R}^{n}$. We precisely investigate the hyperplane arrangement associated to the permutation group $\mathfrak{S}_{n}$ of $n$ elements defined by

$$
\mathcal{A}_{n}:=\left\{H_{i, j} \mid i, j \in[n], i<j\right\} \quad \text { with } \quad H_{i, j}=\left\{x \in \mathbb{R}^{n} \mid x_{i}=x_{j}\right\} .
$$

The set formed by the chambers of $\mathcal{A}_{n}$ is

$$
C_{\mathcal{A}_{n}}=\left\{C_{\sigma} \mid \sigma \in \mathfrak{S}_{n}\right\} \quad \text { with } \quad C_{\sigma}:=\left\{x \in \mathbb{R}^{n} \mid x_{\sigma(1)}<x_{\sigma(2)}<\cdots<x_{\sigma(n)}\right\} .
$$


For $i, j \in[n]$ with $i \neq j$, assign the variable $q_{i, j}$ to the half-space $\left\{x \in \mathbb{R}^{n} \mid x_{i}<x_{j}\right\}$. On $\mathcal{A}_{n}$, the ring $R_{\mathcal{A}_{n}}:=\mathbb{Z}\left[q_{i, j} \mid i, j \in[n]\right]$ and the module $M_{\mathcal{A}_{n}}:=\left\{\sum_{\sigma \in \mathfrak{S}_{n}} x_{\sigma} C_{\sigma} \mid x_{\sigma} \in R_{\mathcal{A}_{n}}\right\}$ are considered. The Aguiar-Mahajan bilinear form becomes $\mathrm{v}_{n}: M_{\mathcal{A}_{n}} \times M_{\mathcal{A}_{n}} \rightarrow R_{\mathcal{A}_{n}}$ defined, for $C_{\sigma}, C_{\tau} \in C_{\mathcal{A}_{n}}$, by

$$
\mathrm{v}_{n}\left(C_{\sigma}, C_{\tau}\right)=\prod_{\substack{\{i, j\} \in\left(\begin{array}{c}
{[n] \\
i<j \\
2}
\end{array}\right) \\
\tau^{-1} \circ \sigma(i)>\tau^{-1} \circ \sigma(j)}} q_{\sigma(i), \sigma(j)},
$$

and $\gamma$ the linear map $\gamma_{n}: M_{\mathcal{A}_{n}} \rightarrow M_{\mathcal{A}_{n}}$ defined, for $C_{\tau} \in C_{\mathcal{A}_{n}}$, by

$$
\gamma_{n}\left(C_{\tau}\right):=\sum_{\sigma \in \mathfrak{S}_{n}} \mathrm{v}_{n}\left(C_{\tau}, C_{\sigma}\right) C_{\sigma}
$$

Proposition 2.1. For an integer $n \geq 2$, we have

$$
\operatorname{det} \gamma_{n}=\prod_{\substack{I \in 2^{[n]} \\
\# I \geq 2}}\left(1-\prod_{\{i, j\} \in\left(\begin{array}{l}
I \\
2
\end{array}\right)} q_{i, j} q_{j, i}\right)^{(\# I-2) !(n-\# I+1) !} .
$$

Proof. We first discuss about the general case of hyperplane arrangements. A flat of $\mathcal{A}$ is an intersection of hyperplanes in $\mathcal{A}$. Denote the set formed by the flats of $\mathcal{A}$ by $L_{\mathcal{A}}$. The weight of a flat $E \in L_{\mathcal{A}}$ is the monomial $\mathrm{b}_{E}:=\prod_{\substack{H \in \mathcal{A} \\ E \subset \boldsymbol{H}}} h_{H}^{+} h_{H}^{-}$, and, if we choose a hyperplane $H$ containing $E$, the multiplicity $\beta_{E}$ of $E$ is half the number of chambers $C \in C_{\mathcal{A}}$ which have the property that $E$ is the minimal edge containing $\bar{C} \cap H$. Aguiar and Mahajan proved that [1, Theorem 8.11]

$$
\operatorname{det} \gamma=\prod_{E \in L_{\mathcal{A}}}\left(1-\mathrm{b}_{E}\right)^{\beta_{E}}
$$

Now, concerning $\mathcal{A}_{n}$, let $L_{\mathcal{A}_{n}}^{\prime}=\left\{E \in L_{\mathcal{A}_{n}} \mid \beta_{E} \neq 0\right\}$. For a subset $I \subseteq[n]$ with $\# I \geq 2$, denote by $E_{I}$ the edge $\bigcap H_{i, j}$. Randriamaro proved that [7, Lemma 3.2, 3.3]

$$
\begin{gathered}
\{i, j\} \in\left(\begin{array}{l}
I \\
2
\end{array}\right) \\
L_{\mathcal{A}_{n}}^{\prime}=\left\{E_{I}|I \subseteq[n],| I \mid \geq 2\right\} \quad \text { and } \quad \beta_{E_{I}}=(\# I-2) !(n-\# I+1) !
\end{gathered}
$$

Take a partition $\lambda=\left(p_{1}, \ldots, p_{k}\right) \in \operatorname{Par}(n)$ of $n$. Denote by $\mathfrak{S}_{\lambda}$ the subgroup $\prod_{i \in[k]} \mathfrak{S}_{\lambda_{i}}$ of $\mathfrak{S}_{n}$, where $\mathfrak{S}_{\lambda_{i}}$ is the permutation group of the set $\left[p_{i}+p_{i-1}+\cdots+p_{1}\right] \backslash\left[p_{i-1}+\cdots+p_{1}\right]$.

Consider the multiset $I_{\lambda}=\{\overbrace{1, \ldots, 1}^{p_{1} \text { times }}, \overbrace{2, \ldots, 2}^{p_{2} \text { times }}, \ldots, \overbrace{k, \ldots, k}^{p_{k} \text { times }}\}$. Denote by $\mathfrak{S}_{I_{\lambda}}$ the permutation set of the multiset $I_{\lambda}$. For $s \in[n]$, define $\dot{s}:=i$ if $s \in\left[p_{i}+p_{i-1}+\cdots+p_{1}\right] \backslash\left[p_{i-1}+\cdots+p_{1}\right]$. Let $\mathrm{p}: \mathfrak{S}_{n} \rightarrow \mathfrak{S}_{I_{\lambda}}$ be the projection $\mathrm{p}(\sigma):=\sigma \dot{(1)} \sigma(2) \ldots \sigma(n)$. For $\dot{\sigma} \in \mathfrak{S}_{I_{\lambda}}$, define the element $C_{\dot{\sigma}}:=\sum_{\sigma \in \mathrm{p}^{-1}(\dot{\sigma})} C_{\sigma} \in M_{\mathcal{A}_{n}}$. Denote by $M_{\mathcal{A}_{n}}^{\lambda}$ the submodule

$$
M_{\mathcal{A}_{n}}^{\lambda}:=\left\{\sum_{\dot{\sigma} \in \mathfrak{S}_{I_{\lambda}}} x_{C_{\dot{\sigma}}} C_{\dot{\sigma}} \mid x_{C_{\dot{\sigma}}} \in R_{\mathcal{A}_{n}}\right\}
$$


For $s, t \in[n]$ with $s \neq t$, assign the variable $q_{\dot{s}, \dot{t}}$ to the half-space $\left\{x \in \mathbb{R}^{n} \mid x_{s}<x_{t}\right\}$.

Proposition 2.2. Let $n \in \mathbb{N}^{*}$, and $\lambda \in \operatorname{Par}(n)$. Then, $\gamma_{n}\left(M_{\mathcal{A}_{n}}^{\lambda}\right)=M_{\mathcal{A}_{n}}^{\lambda}$.

Proof. If $\sigma \in \mathfrak{S}_{n}$ such that $\mathrm{p}(\sigma)=\dot{\sigma} \in \mathfrak{S}_{I_{\lambda}}$, then $\mathrm{p}^{-1}(\dot{\sigma})=\mathfrak{S}_{\lambda} \sigma$. Let $v \sigma \in \mathfrak{S}_{\lambda} \sigma$, and $\dot{\tau} \in \mathfrak{S}_{I_{\lambda}}$. If $\mathrm{p}(\tau)=\dot{\tau}$,

$$
\begin{aligned}
& \mathrm{v}_{n}\left(C_{\dot{\tau}}, C_{v \sigma}\right)=\sum_{\varphi \in \mathfrak{S}_{\lambda}} \mathrm{v}_{n}\left(C_{\varphi \tau}, C_{v \sigma}\right) \\
& =\sum_{\varphi \in \mathfrak{S}_{\lambda}} \prod_{\substack{\{i, j\} \in\left(\begin{array}{c}
n n] \\
i<j \\
2
\end{array}\right) \\
\sigma^{-1} v^{-1} \circ \varphi \tau(i)>\sigma^{-1} v^{-1} \circ \varphi \tau(j)}} q_{\varphi \dot{\tau}(i), \varphi \dot{\tau}(j)}
\end{aligned}
$$

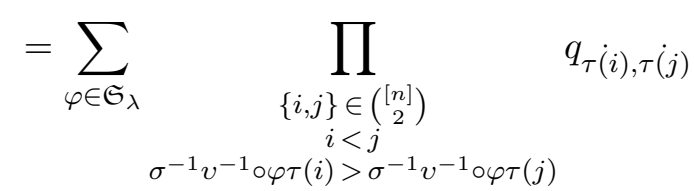

$$
\begin{aligned}
& =\sum_{\varphi \in \mathfrak{S}_{\lambda}} \prod_{\substack{\{i, j\} \in\left(\begin{array}{c}
{[n] \\
i<j \\
2}
\end{array}\right) \\
q_{\tau(i), \tau(j)}}} q \\
& =\mathrm{v}_{n}\left(C_{\dot{\tau}}, C_{\sigma}\right) \text {. }
\end{aligned}
$$

Hence,

$$
\begin{aligned}
\gamma_{n}\left(C_{\dot{\tau}}\right) & =\sum_{\sigma \in \mathfrak{S}_{n}} \mathrm{v}_{n}\left(C_{\dot{\tau}}, C_{\sigma}\right) C_{\sigma} \\
& =\sum_{\dot{\sigma} \in \mathfrak{S}_{\lambda_{\lambda}}} \sum_{v \in \mathfrak{S}_{\lambda}} \mathrm{v}_{n}\left(C_{\dot{\tau}}, C_{v \sigma}\right) C_{v \sigma} \\
& =\sum_{\dot{\sigma} \in \mathfrak{S}_{I_{\lambda}}} \sum_{v \in \mathfrak{S}_{\lambda}} \mathrm{v}_{n}\left(C_{\dot{\tau}}, C_{\sigma}\right) C_{v \sigma} \\
& =\sum_{\dot{\sigma} \in \mathfrak{S}_{I_{\lambda}}} \mathrm{v}_{n}\left(C_{\dot{\tau}}, C_{\sigma}\right) \sum_{v \in \mathfrak{S}_{\lambda}} C_{v \sigma} \\
& =\sum_{\dot{\sigma} \in \mathfrak{S}_{I_{\lambda}}} \mathrm{v}_{n}\left(C_{\dot{\tau}}, C_{\sigma}\right) C_{\dot{\sigma}} .
\end{aligned}
$$

\section{The Bra-Ket Product on $\mathbf{H}$}

We prove some useful properties of the map in Theorem 1.3 . We particularly connect it to the bilinear form $\mathrm{v}_{n}$ defined in Section 2 .

Lemma 3.1. The vector space generated by our particle states is

$$
\mathbf{H}=\left\{\sum_{i=1}^{n} \mu_{i} \mathrm{~b}_{i} \mid n \in \mathbb{N}^{*}, \mu_{i} \in \mathbb{C}\left[q_{i, j}\right], \mathrm{b}_{i} \in \mathbf{B}\right\} .
$$


Proof. Let $i \in \mathbb{N}^{*}$. We have,

$$
\begin{aligned}
\mathrm{a}_{i} \mathrm{a}_{j_{1}}^{\dagger} \ldots \mathrm{a}_{j_{t}}^{\dagger}= & q_{i, j_{1}} \ldots q_{i, j_{t}} \mathrm{a}_{j_{1}}^{\dagger} \ldots \mathrm{a}_{j_{t}}^{\dagger} \mathrm{a}_{i} \\
& +\sum_{\substack{u \in[t] \\
j_{u}=i}} q_{i, j_{1}} \ldots q_{i, j_{u-1}} \mathrm{a}_{j_{1}}^{\dagger} \ldots \widehat{\mathrm{a}_{j_{u}}^{\dagger}} \ldots \mathrm{a}_{j_{t}}^{\dagger},
\end{aligned}
$$

where the hat over the $u^{\text {th }}$ term of the product indicates that this term is omitted. So

$$
\mathrm{a}_{i} \mathrm{a}_{j_{1}}^{\dagger} \ldots \mathrm{a}_{j_{t}}^{\dagger}|0\rangle=\sum_{\substack{u \in[t] \\ j_{u}=i}} q_{i, j_{1}} \ldots q_{i, j_{u-1}} \mathrm{a}_{j_{1}}^{\dagger} \ldots \widehat{\mathrm{a}_{j_{u}}^{\dagger}} \ldots \mathrm{a}_{j_{t}}^{\dagger}|0\rangle .
$$

Thus, one can recursively remove every annihilation operator $\mathbf{a}_{i}$ of an element $\mathbf{a}|0\rangle \in \mathbf{H}$.

Lemma 3.2. Let $\left(i_{1}, \ldots, i_{s}\right) \in\left(\mathbb{N}^{*}\right)^{s}$ and $\left(j_{1}, \ldots, j_{t}\right) \in\left(\mathbb{N}^{*}\right)^{t}$. If, as multisets, $\left\{i_{1}, \ldots, i_{s}\right\}$ is different from $\left\{j_{1}, \ldots, j_{t}\right\}$, then $\left\langle 0\left|\mathrm{a}_{i_{s}} \ldots \mathrm{a}_{i_{1}} \mathrm{a}_{j_{1}}^{\dagger} \ldots \mathrm{a}_{j_{t}}^{\dagger}\right| 0\right\rangle=0$.

Proof. Suppose that $v$ is the smallest integer in $[s]$ such that $i_{v} \notin\left\{j_{1}, \ldots, j_{t}\right\} \backslash\left\{i_{1}, \ldots, i_{v-1}\right\}$. Then

$$
\mathrm{a}_{i_{s}} \ldots \mathrm{a}_{i_{1}} \mathrm{a}_{j_{1}}^{\dagger} \ldots \mathrm{a}_{j_{t}}^{\dagger}=P \mathrm{a}_{i_{v}} \ldots \mathrm{a}_{i_{1}}+Q \mathrm{a}_{i_{v}} \text { with } P, Q \in \mathbf{A} .
$$

We deduce that $\mathrm{a}_{i_{s}} \ldots \mathrm{a}_{i_{1}} \mathrm{a}_{j_{1}}^{\dagger} \ldots \mathrm{a}_{j_{t}}^{\dagger}|0\rangle=P \mathrm{a}_{i_{v}} \ldots \mathrm{a}_{i_{1}}|0\rangle+Q \mathrm{a}_{i_{v}}|0\rangle=0$.

Similarly, suppose that $u$ is the smallest integer in $[t]$ such that $j_{u}$ does not belong to the multiset $\left\{i_{1}, \ldots, i_{s}\right\} \backslash\left\{j_{1}, \ldots, j_{u-1}\right\}$. Then

$$
\mathrm{a}_{i_{s}} \ldots \mathrm{a}_{i_{1}} \mathrm{a}_{j_{1}}^{\dagger} \ldots \mathrm{a}_{j_{t}}^{\dagger}=\mathrm{a}_{j_{1}}^{\dagger} \ldots \mathrm{a}_{j_{u}}^{\dagger} P^{\prime}+\mathrm{a}_{j_{u}}^{\dagger} Q^{\prime} \quad \text { with } \quad P^{\prime}, Q^{\prime} \in \mathbf{A} .
$$

And $\langle 0| \mathrm{a}_{i_{s}} \ldots \mathrm{a}_{i_{1}} \mathrm{a}_{j_{1}}^{\dagger} \ldots \mathrm{a}_{j_{t}}^{\dagger}=\langle 0| \mathrm{a}_{j_{1}}^{\dagger} \ldots \mathrm{a}_{j_{u}}^{\dagger} P^{\prime}+\langle 0| \mathrm{a}_{j_{u}}^{\dagger} Q^{\prime}=0$.

Therefore, we just need to investigate the product $\left\langle 0\left|\mathrm{a}_{i_{n}} \ldots \mathrm{a}_{i_{1}} \mathrm{a}_{j_{1}}^{\dagger} \ldots \mathrm{a}_{j_{n}}^{\dagger}\right| 0\right\rangle$ where $\left(j_{1}, \ldots, j_{n}\right)$ is a multiset permutation of $\left(i_{1}, \ldots, i_{n}\right)$.

Lemma 3.3. Let $\sigma, \tau \in \mathfrak{S}_{n}$, and $C_{\sigma}, C_{\tau} \in C_{\mathcal{A}_{n}}$ their associated chambers. Then,

$$
\left\langle 0\left|\mathrm{a}_{\sigma(n)} \ldots \mathrm{a}_{\sigma(1)} \mathrm{a}_{\tau(1)}^{\dagger} \ldots \mathrm{a}_{\tau(n)}^{\dagger}\right| 0\right\rangle=\mathrm{v}_{n}\left(C_{\sigma}, C_{\tau}\right) .
$$

Proof. We have

$$
\begin{aligned}
\left\langle 0\left|\mathrm{a}_{\sigma(n)} \ldots \mathrm{a}_{\sigma(1)} \mathrm{a}_{\tau(1)}^{\dagger} \ldots \mathrm{a}_{\tau(n)}^{\dagger}\right| 0\right\rangle= & \prod_{s \in[n]} \prod_{\substack{t \in[n] \backslash[s] \\
\tau^{-1} \circ \sigma(s)>\tau^{-1} \circ \sigma(t)}} q_{\sigma(s), \sigma(t)} \\
= & \prod_{\substack{\{s, t\} \in\left(\begin{array}{c}
{[n] \\
s<t \\
2}
\end{array}\right) \\
\tau^{-1} \circ \sigma(s)>\tau^{-1} \circ \sigma(t)}} q_{\sigma(s), \sigma(t)} \\
= & \mathrm{v}_{n}\left(C_{\sigma}, C_{\tau}\right) .
\end{aligned}
$$

For $s, t \in[n]$ with $s \neq t$, assign the variable $q_{\dot{s}, \dot{t}}$ to the half-space $\left\{x \in \mathbb{R}^{n} \mid x_{s}<x_{t}\right\}$. 
Lemma 3.4. Let $\lambda \in \operatorname{Par}(\mathrm{n})$, and $\dot{\sigma}, \dot{\tau} \in \mathfrak{S}_{I_{\lambda}}$. Then, for every $\tau \in \mathrm{p}^{-1}(\dot{\tau})$,

$$
\left\langle 0\left|\mathrm{a}_{\dot{\sigma}(n)} \ldots \mathrm{a}_{\dot{\sigma}(1)} \mathrm{a}_{\dot{\tau}(1)}^{\dagger} \ldots \mathrm{a}_{\dot{\tau}(n)}^{\dagger}\right| 0\right\rangle=\mathrm{v}_{n}\left(C_{\dot{\sigma}}, C_{\tau}\right) .
$$

Proof. We have

$$
\begin{aligned}
& \left\langle 0\left|\mathrm{a}_{\dot{\sigma}(n)} \ldots \mathrm{a}_{\dot{\sigma}(1)} \mathrm{a}_{\dot{\tau}(1)}^{\dagger} \ldots \mathrm{a}_{\dot{\tau}(n)}^{\dagger}\right| 0\right\rangle=\sum_{\substack{v \in \mathfrak{S}_{n} \\
\dot{\sigma}=\dot{\tau} \circ v}} \prod_{s \in[n]} \prod_{\substack{t \in[n] \backslash[s] \\
v(s)>v(t)}} q_{\dot{\sigma}(s), \dot{\tau} \circ v(t)}
\end{aligned}
$$

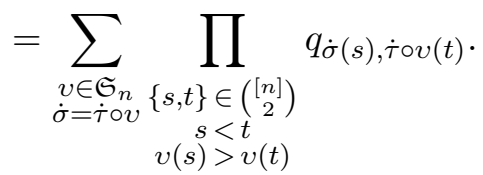

For every $\sigma \in \mathrm{p}^{-1}(\dot{\sigma})$, and $\tau \in \mathrm{p}^{-1}(\dot{\tau})$, we have, on one side,

$$
\dot{\sigma}=\dot{\tau} \circ v \Longleftrightarrow \mathfrak{S}_{\lambda} \sigma=\mathfrak{S}_{\lambda} \tau v \Longleftrightarrow v \in \tau^{-1} \mathfrak{S}_{\lambda} \sigma .
$$

On the other side, if id is the identity permutation, there exists $\varphi \in \mathfrak{S}_{\lambda}$ such that $v=\tau^{-1} \varphi \sigma$, and

$$
\dot{\tau} \circ v(t)=\dot{\tau} \circ \tau^{-1} \varphi \sigma(t)=\dot{\mathrm{id}} \circ \varphi \sigma(t)=\dot{\mathrm{id}} \circ \sigma(t)=\dot{\sigma}(t) .
$$

Then,

$$
\begin{aligned}
\left\langle 0\left|\mathrm{a}_{\dot{\sigma}(n)} \ldots \mathrm{a}_{\dot{\sigma}(1)} \mathrm{a}_{\dot{\tau}(1)}^{\dagger} \ldots \mathrm{a}_{\dot{\tau}(n)}^{\dagger}\right| 0\right\rangle & =\sum_{\varphi \in \mathfrak{S}_{\lambda}} \prod_{\begin{array}{c}
\{s, t\} \in\left(\begin{array}{c}
{[n]} \\
s< \\
s<t \\
\tau^{-1} \varphi \sigma(s)>\tau^{-1} \varphi \sigma(t) \\
\end{array}\right. \\
\end{array}} q_{\dot{\sigma}(s), \dot{\sigma}(t)} \\
& =\mathrm{v}_{n}\left(C_{\dot{\sigma}}, C_{\tau}\right)
\end{aligned}
$$

\section{The Conjecture of Zagier}

To prove the extended Zagier's conjecture, we first have to return to the general case of hyperplane arrangements. The set $F_{\mathcal{A}}$ is a monoid with product $F G$ defined, for $F, G \in F_{\mathcal{A}}$, by

$$
\epsilon_{H}(F G)= \begin{cases}\epsilon_{H}(F) & \text { if } \epsilon_{H}(F) \neq 0, \\ \epsilon_{H}(G) & \text { otherwise. }\end{cases}
$$

It is also a meet-semilattice with partial order $\preceq$ defined, for $F, G \in F_{\mathcal{A}}$, by

$$
F \preceq G \Longleftrightarrow \epsilon_{H}(F)=\epsilon_{H}(G) \text { whenever } \epsilon_{H}(F) \neq 0 \text {. }
$$

Denote by $O$ the face in $F_{\mathcal{A}}$ such that, for every $H \in \mathcal{A}, \epsilon_{H}(O)=0$. The rank of a face $F \in F_{\mathcal{A}}$ is $\operatorname{rk} F:=\operatorname{dim} \bigcap_{A} H-\operatorname{dim} O$. The weight of a face $F \in F_{\mathcal{A}}$ is the monomial $\underset{F \subseteq \mathcal{A}}{H \subseteq H}$

$\mathrm{b}_{F}:=\prod_{\substack{H \in \mathcal{A} \\ F \subseteq H}} h_{H}^{+} h_{H}^{-}$. Clearly, $\mathrm{b}_{F}=0$ if $F \in C_{\mathcal{A}}$ 
A nested face is a pair $(F, G)$ of faces in $F_{\mathcal{A}}$ such that $F \prec G$. For a nested face $(F, G)$, define the set $F_{\mathcal{A}}^{(F, G)}:=\left\{K \in F_{\mathcal{A}} \mid F \preceq K \preceq G\right\}$, and the polynomial $\Delta_{F, G}:=\prod_{K \in F_{\mathcal{A}}^{(F, G)}}\left(1-\mathrm{b}_{K}\right)$.

We prove a variant result of Aguiar and Mahajan [1, Proposition 8.13].

Proposition 4.1. Let $\mathcal{A}$ be a hyperplane arrangement in $\mathbb{R}^{n}$. Each entry of $\gamma^{-1}: M_{\mathcal{A}} \rightarrow M_{\mathcal{A}}$ is an element of $\bigcup_{C \in C_{\mathcal{A}}} \frac{R_{\mathcal{A}}}{\Delta_{O, C}}$.

Proof. As det $\gamma$ is a polynomial in $R_{\mathcal{A}}$ with constant term $1, \gamma$ is consequently invertible. For a chamber $D \in C_{\mathcal{A}}$, and a nested face $(A, D)$, let $\mathrm{m}(A, D):=\sum_{\substack{C \in C_{\mathcal{A}} \\ A C=D}} \mathrm{v}(D, C) C$. We prove by backward induction that $\mathrm{m}(A, D)=\sum_{C \in C_{\mathcal{A}}} x_{C} \gamma(C)$ with $x_{C} \in \frac{R_{\mathcal{A}}}{\Delta_{A, D}}$.

We have $\mathrm{m}(D, D)=\gamma(D)$. The opposite of a face $F \in F_{\mathcal{A}}$ is the face $\tilde{F}$ such that, for every $H \in \mathcal{A}, \epsilon_{H}(\tilde{F})=-\epsilon_{H}(F)$. One can read in the proof of [1, Proposition 8.13] that

$$
\mathrm{m}(A, D)-(-1)^{\mathrm{rk} D-\mathrm{rk} A} \mathrm{v}(D, A \tilde{D}) \mathrm{m}(A, A \tilde{D})=\sum_{F \in F_{\mathcal{A}}^{(A, D)} \backslash\{A\}}(-1)^{\mathrm{rk} F-\mathrm{rk} A+1} \mathrm{~m}(F, D) .
$$

By induction hypothesis, for every $C \in C_{\mathcal{A}}$, there exists $a_{C} \in \bigcup_{F \in F_{\mathcal{A}}^{(A, D)} \backslash\{A\}} \frac{R_{\mathcal{A}}}{\Delta_{F, D}}$, such that

$$
\sum_{F \in F_{\mathcal{A}}^{(A, D)} \backslash\{A\}}(-1)^{\mathrm{rk} F-\mathrm{rk} A+1} \mathrm{~m}(F, D)=\sum_{C \in C_{\mathcal{A}}} a_{C} \gamma(C) .
$$

Remark that, for every $F \in F_{\mathcal{A}}^{(A, D)}$, we have $\mathrm{b}_{F}=\mathrm{b}_{A \tilde{F}}$, which means that

$$
\bigcup_{F \in F_{\mathcal{A}}^{(A, A \tilde{D})} \backslash\{A\}} \frac{R_{\mathcal{A}}}{\Delta_{F, A \tilde{D}}}=\bigcup_{F \in F_{\mathcal{A}}^{(A, D)} \backslash\{A\}} \frac{R_{\mathcal{A}}}{\Delta_{F, D}} .
$$

Since $A \leq A \tilde{D}$ and $A(\widetilde{A \tilde{D}})=D$, replacing $D$ with $A \tilde{D}$, there exists also $e_{C} \in \bigcup_{F \in F_{A}^{(A, D)} \backslash\{A\}} \frac{R_{\mathcal{A}}}{\Delta_{F, D}}$, such that, for every $C \in C_{\mathcal{A}}, \mathrm{m}(A, A \tilde{D})-(-1)^{\mathrm{rk} A \tilde{D}-\mathrm{rk} A} \mathrm{v}(A \tilde{D}, D) \mathrm{m}(A, D)=\sum_{C \in C_{\mathcal{A}}} e_{C} \gamma(C)$. Therefore,

$$
\begin{aligned}
\mathrm{m}(A, D)-(-1)^{\mathrm{rk} D-\mathrm{rk} A} \mathrm{v}(D, A \tilde{D}) \mathrm{m}(A, A \tilde{D}) & =\sum_{C \in C_{\mathcal{A}}} a_{C} \gamma(C) \\
\mathrm{m}(A, D)-\mathrm{v}(D, A \tilde{D}) \mathrm{v}(A \tilde{D}, D) \mathrm{m}(A, D) & =\sum_{C \in C_{\mathcal{A}}}\left(a_{C}+(-1)^{\mathrm{rk} D-\mathrm{rk} A} \mathrm{v}(D, A \tilde{D}) e_{C}\right) \gamma(C) \\
\mathrm{m}(A, D) & =\sum_{C \in C_{\mathcal{A}}} \frac{a_{C}+(-1)^{\mathrm{rk} D-\mathrm{rk} A} \mathrm{v}(D, A \tilde{D}) e_{C}}{1-\mathrm{b}_{A}} \gamma(C),
\end{aligned}
$$


with $\frac{a_{C}+(-1)^{\mathrm{rk} D-\mathrm{rk} A} \mathrm{v}(D, A \tilde{D}) e_{C}}{1-\mathrm{b}_{A}} \in \bigcup_{F \in F_{\mathcal{A}}^{(A, D)}} \frac{R_{\mathcal{A}}}{\Delta_{F, D}}=\frac{R_{\mathcal{A}}}{\Delta_{A, D}}$.

For every $D \in C_{\mathcal{A}}$, we have $\mathrm{m}(O, D)=D$. Thus $D=\sum_{C \in C_{\mathcal{A}}} x_{C} \gamma(C)$ with $x_{C} \in \frac{R_{\mathcal{A}}}{\Delta_{O, D}}$, and $\gamma^{-1}(D)=\sum_{C \in C_{\mathcal{A}}} x_{C} C$. Finally, each entry of $\gamma^{-1}$ is an element of $\bigcup_{C \in C_{\mathcal{A}}} \frac{R_{\mathcal{A}}}{\Delta_{O, C}}$.

We can deduce the extended Zagier's conjecture.

Corollary 4.2. Let $n \geq 2$, and suppose that $q_{i, j}=q_{j, i}=q$. Then each entry of $\gamma_{n}^{-1}$ is an element of $\frac{\mathbb{Z}[q]}{\prod_{i \in[n-1]}\left(1-q^{i^{2}+i}\right)^{n-i}}$.

Proof. Let $O_{n}=\bigcap_{H \in \mathcal{A}_{n}} H, C_{\sigma} \in C_{\mathcal{A}_{n}}$, and $i, j \in[n-1]$ such that $i \geq j$. A $i$-dimensional face $F_{i} \in F_{\mathcal{A}_{n}}^{\left(O_{n}, C_{\sigma}\right)}$ has the form

$$
F_{i}=\{x \in \mathbb{R}^{n} \mid x_{\sigma(1)}<\cdots<\overbrace{x_{\sigma(j)}=x_{\sigma(j+1)}=\cdots=x_{\sigma(j+n-i+1)}}^{n-i+1 \text { variables }}<\cdots<x_{\sigma(n)}\} .
$$

Thus

$$
\mathrm{b}_{F_{i}}=\prod_{\{k, l\} \in\left(\begin{array}{c}
I_{i} \\
2
\end{array}\right)} q_{k, l} q_{l, k} \quad \text { with } \quad I_{i}=\{\sigma(j), \sigma(j+1), \ldots, \sigma(j+n-i+1)\} .
$$

If $q=q_{k, l}=q_{l, k}$, then $\mathrm{b}_{F_{i}}=q^{(n-i)^{2}+(n-i)}$. Since $F_{\mathcal{A}_{n}}^{\left(O_{n}, C_{\sigma}\right)}$ contains exactly $i$-dimensional face, we get $\Delta_{O_{n}, C_{\sigma}}=\prod_{i \in[n-1]}\left(1-q^{(n-i)^{2}+(n-i)}\right)^{i}$. Finally, we deduce from Proposition 4.1 that each entry of $\gamma_{n}^{-1}$ is an element of $\frac{\mathbb{Z}[q]}{\prod_{i \in[n-1]}\left(1-q^{(n-i)^{2}+(n-i)}\right)^{i}}$. 


\section{References}

[1] M. Aguiar, S. Mahajan, Topics in Hyperplane Arrangements, Mathematical Surveys and Monographs 226, 2017.

[2] M. Bozejko, R. Speicher, Completely Positive Maps on Coxeter Groups, Deformed Commutation Relations, and Operator Spaces, Math. Ann. (300) (1994), 97-120.

[3] G. Duchamp, A. Klyachko, D. Krob, J.-Y. Thibon, Noncommutative Symmetric Functions III: Deformations of Cauchy and Convolution Algebras, Discrete Math. Theor. Comput. Sci. (1) (1997), 159-216.

[4] O. Greenberg, Example of Infinite Statistics, Phys. Rev. Lett. (64) 7 (1990), 705-708.

[5] O. Greenberg, Particles with small Violations of Fermi or Bose Statistics, Phys. Rev. D (43) 12 (1991), 4111-4120.

[6] S. Meljanac, D. Svrtan, Study of Gram Matrices in Fock Representation of Multiparametric Canonical Commutation Relations, extended Zagier's Conjecture, Hyperplane Arrangements and Quantum Groups, Math. Commun. (1) 1 (1996), 1-24.

[7] H. Randriamaro, Computing the Varchenko Determinant of a Bilinear Form, Irish Math. Soc. Bulletin (82) (2018), 79-90.

[8] R. Speicher, Generalized Statistics of Macroscopic Fields, Lett. Math. Phys. (27) (1993), 97-104.

[9] D. Zagier, Realizability of a Model in Infinite Statistics, Comm. Math. Phys. (147) (1992), 199-210. 